\title{
PET/CT Enterography in Crohn Disease: Correlation of Disease Activity on CT Enterography with ${ }^{18}$ F-FDG Uptake
}

\author{
David Groshar ${ }^{1-3}$, Hanna Bernstine ${ }^{1,3}$, Dorit Stern ${ }^{1,2}$, Jacob Sosna ${ }^{4,5}$, Merab Eligalashvili ${ }^{1}$, Evren G. Gurbuz ${ }^{4}$, \\ Yaron $\mathrm{Niv}^{3,6}$, and Gerald Fraser ${ }^{3,6}$ \\ ${ }^{1}$ Department of Nuclear Medicine, Rabin Medical Center, Petah Tikva, Israel; ${ }^{2}$ Department of Nuclear Medicine, Assuta Medical \\ Center, Tel-Aviv, Israel; ${ }^{3}$ Sackler Faculty of Medicine, Tel Aviv University, Tel Aviv, Israel; ${ }^{4}$ Department of Radiology, Hadassah \\ Hebrew University Medical Center, Jerusalem, Israel; ${ }^{5}$ Department of Radiology, Beth Israel Deaconess Medical Center, Harvard \\ Medical School, Boston, Massachusetts; and ${ }^{6}$ Department of Gastroenterology, Rabin Medical Center, Petah Tikva, Israel
}

We combined ${ }^{18}$ F-FDG PET and CT enterography in a single examination and compared the level of ${ }^{18} \mathrm{~F}-\mathrm{FDG}$ uptake measured by maximal standardized uptake value (SUVmax) with the CT enterography patterns of disease activity found in patients with Crohn disease (CD). Methods: Twenty-eight patients (mean age, $37.5 \mathrm{y} ; 11$ male and 17 female) suspected of having active CD underwent PET/CT enterography. Abnormal bowel segments recognized on $\mathrm{CT}$ enterography were graded qualitatively for the presence of perienteric fat infiltration, the comb sign, and intramural attenuation and by quantitative measurements of mural enhancement (Hounsfield units) and thickness ( $\mathrm{mm})$. Also, for each patient, normal segments of the ileum and colon were noted, and CT enterography measurements of thickness and enhancement were obtained. For segments detected on CT enterography, a volume of interest was placed on the fused ${ }^{18} \mathrm{~F}-\mathrm{FDG}$ PET scan, and the SUVmax was obtained. Results: Of the 28 patients with suspected active $C D, 22$ had 85 abnormal segments and 6 had no abnormal segments. SUVmax was significantly higher in the abnormal segments than in the normal segments (5.0 \pm 2.5 [95\% confidence interval, 4.5-5.5] and $2.1 \pm 0.69$ [95\% confidence interval, 1.9-2.2], respectively; $P<0.0001$ ). A good correlation was found between SUVmax with CT enterography measurements of mural thickness and enhancement $(P<$ $0.00001)$. There was a significant difference in SUVmax between the 3 levels of disease activity found by intramural attenuation, perienteric fat infiltration, and the comb sign on CT enterography. SUVmax was significantly higher when there were intense CT enterography findings of active disease $(P<0.001)$. Conclusion: SUVmax assessment may allow an objective, reliable indication of the grade and severity of inflammation activity in abnormal segments of the bowel detected by CT enterography.

Key Words: correlative imaging; gastroenterology; PET/CT; Crohn disease; FDG

J Nucl Med 2010; 51:1009-1014

DOI: 10.2967/jnumed.109.073130

Received Nov. 24, 2009; revision accepted Mar. 4, 2010.

For correspondence or reprints contact: David Groshar, Department of Nuclear Medicine, Rabin Medical Center, Jabotinsky St., Petah Tikva, Israel 49100.

E-mail: davidgr2@clalit.org.il

COPYRIGHT $\odot 2010$ by the Society of Nuclear Medicine, Inc.
C rohn disease (CD) is a chronic granulomatous inflammatory disease involving the gastrointestinal tract with a tendency toward remission and relapse. CD is usually found as discontinuous, segmental, often multifocal inflammation of the small intestine and colon, but any portion of the gastrointestinal tract from mouth to anus may be involved. Most commonly, in nearly half of patients, $\mathrm{CD}$ is seen in both the small and the large bowel $(1,2)$.

The initial diagnosis of $\mathrm{CD}$ and the evaluation of disease activity are based on a combination of clinical symptoms, physical findings, laboratory investigations, endoscopy, and imaging tests. No single diagnostic test allows a definite diagnosis of the presence, severity, and extent of disease; the inflammatory lesion activity; and the presence of extraintestinal complications $(3,4)$.

CT enterography is used to detect enteric inflammation and extraenteric complications. CT enterography differs from routine abdominal $\mathrm{CT}$ in that the patient drinks large volumes of a neutral oral contrast agent that allows better delineation of the bowel wall and lumen. Morphologic and pathophysiologic patterns on CT enterography such as mural thickness, mural enhancement, mural stratification, increased attenuation of perienteric fat, and pericolic or perienteric hypervascularity (the comb sign) have been reported to indicate active inflammatory CD (3-5).

Whole-body ${ }^{18}$ F-FDG PET/CT is now a standard practice in the evaluation of patients presenting with cancer and provides important information about metabolism and anatomy. The PET part of this hybrid modality relies on the accumulation of ${ }^{18} \mathrm{~F}-\mathrm{FDG}$ in metabolically active cells. ${ }^{18} \mathrm{~F}$-FDG accumulates in malignant tissues but also at sites of infection and inflammation and in autoimmune and granulomatous diseases by the overexpression of distinct facultative glucose transporter isotypes (mainly glucose transporters 1 and 3 ) and by an overproduction of glycolytic enzymes in cancer cells and inflammatory cells (6). Standardized uptake value (SUV), representing glucose 
metabolic activity, is an objective measurement of ${ }^{18} \mathrm{~F}-\mathrm{FDG}$ uptake in tissues and is typically higher in areas of inflammation than in normal tissue. ${ }^{18} \mathrm{~F}-\mathrm{FDG}$ PET has been shown to be both sensitive and specific in the detection of active sites of disease within both the small bowel and the colon and to correlate well with the clinical, endoscopic, and biologic activity of CD (7-12).

By combining the morphologic and physiologic patterns obtained by CT enterography with the ${ }^{18} \mathrm{~F}-\mathrm{FDG}$ metabolic activity obtained by PET, PET/CT enterography as a single test may provide accurately fused morphologic, physiologic, and metabolic imaging that can be useful in the diagnosis, follow-up, and objective assessment of CD. Our hypothesis was that SUV activity would be related to CT enterography parameters, enabling quantification of $\mathrm{CD}$ activity. In this pilot study, we compared the metabolic activity of ${ }^{18} \mathrm{~F}-\mathrm{FDG}$ obtained by SUV measurements with patterns of disease activity obtained by CT enterography.

\section{MATERIALS AND METHODS}

This study was approved by the review board of our institution, and written informed consent was obtained from all participants.

The study included patients who were known to have or suspected of having active $\mathrm{CD}$, had been scheduled for CT enterography ordered as part of routine clinical practice by a gastroenterologist, and consented prospectively to undergo PET/CT enterography instead. All patients were examined in the inflammatory bowel disease clinic of a tertiary referral center (Beilinson Hospital, Petah Tikva, Israel) by a single gastroenterologist with $26 \mathrm{y}$ of experience. Patients who were younger than $18 \mathrm{y}$, pregnant, or unable to receive iodinated contrast material (allergy, renal insufficiency) were excluded.

Twenty-eight consecutive patients (mean age, $37.5 \mathrm{y}$; range, 18.6-70.7 y; 11 male [mean age, 42.1 y; range, 18.6-70.7 y] and 17 female [mean age, $34.5 \mathrm{y}$; range, 19.6-65.6 y]) were recruited between September 2008 and September 2009. The study was performed to assess disease activity in 20 patients who had established CD with suspected stricture of the small intestine based on appropriate clinical symptoms or who had established but asymptomatic $\mathrm{CD}$ with laboratory findings compatible with active disease such as elevated C-reactive protein and erythrocyte sedimentation rate, thrombocytosis, refractory iron-deficiency anemia, and low albumin. In a further 8 patients, CD was strongly suspected on the basis of clinical and laboratory findings but colonoscopy was negative for disease.

\section{${ }^{18}$ F-FDG PET/CT Enterography Scans}

Patients fasted for a minimum of $4 \mathrm{~h}$ before the intravenous injection of $185 \mathrm{MBq}(5 \mathrm{mCi})$ of ${ }^{18} \mathrm{~F}-\mathrm{FDG}$. For the CT enterography portion of the study, a large volume of bowel-distending negative contrast material was administered (13). A 1,250-mL volume of the solution, which comprised $1,000 \mathrm{~mL}$ of water and $250 \mathrm{~mL}$ of lactulose $(670 \mathrm{mg} / \mathrm{mL})$ (Laevolac; Fresenius-Kabi), was ingested orally over $40-50 \mathrm{~min}$ at a steady rate. All images were obtained from the mid thorax through the pelvis using an integrated 8-slice PET/CT scanner (Discovery ST; GE Healthcare). The parameters for CT image acquisition were as follows: helical imaging, $120 \mathrm{kV}$, modulated 80-280 mAs, 2.5-mm slice thickness, 0.875 pitch, and image reconstruction every $2.5 \mathrm{~mm}$. Iodine contrast medium (100 $\mathrm{mL}$ of Ultravist 300 [iopromide, $0.623 \mathrm{~g} / \mathrm{mL}$ ]; Bayer Schering Pharma AG) was intravenously administered at a rate of $4.0 \mathrm{~mL} / \mathrm{s}$ using an automatic power injector (Dual-Shot; Nemoto), with scanning initiated after a 40-s delay. Immediately after CT, a PET scan was acquired in 3-dimensional mode at a rate of 120-150 s per bed position. CT data were used for attenuation correction. Projection data were reconstructed online with manufacturer-supplied 3-dimensional iterative software (VuePoint 3-D; 2 iterations, 28 subsets, 3.27-mm slice thickness).

\section{Selection of Bowel Segments for Analysis}

A gastrointestinal radiologist with $30 \mathrm{y}$ of clinical expertise in body CT, and 2 nuclear medicine experts with 5 and 3 y of expertise in PET/CT, reviewed all the images. For each patient, the radiologist first visually assessed the gastrointestinal tract from the stomach to the anus on CT enterograms while unaware of the PET findings. Any 5 -cm or longer bowel segment showing mural hyperenhancement or mural stratification was noted as abnormal. Mural hyperenhancement was defined as a bowel wall segment showing increased attenuation, compared with adjacent normal bowel wall, and mural stratification was defined as 2 or 3 layers within the bowel wall. Then, for each abnormal segment noted, visual qualitative assessment of perienteric fat infiltration (Supplemental Fig. 1) and the comb sign (Supplemental Fig. 2) was categorized as absent, moderate, or intense; intramural attenuation was categorized as fat, soft-tissue, or water (Fig. 1-3); and quantitative measurements of mural enhancement (Hounsfield units [HU]) and mural thickness $(\mathrm{mm})$ were obtained using the largest region of interest possible. Also, for each patient, 1 normal segment of the ileum and 1 normal segment of the colon were noted and CT enterography measurements of mural thickness and mural enhancement obtained.

For each abnormal and normal segment detected on CT enterography, a 3-dimensional volume-of-interest box was created on the axial slice of the fused ${ }^{18} \mathrm{~F}-\mathrm{FDG}$ PET scan and manually adjusted to encompass the respective bowel segment in all $(x, y$,
FIGURE 1. Intramural water attenuation on PET/CT enterography. (A) Coronal PET image shows increased uptake of ${ }^{18} \mathrm{~F}-\mathrm{FDG}$ in distal ileum (SUVmax, 7). (B) Fused PET/CT enterogram shows ${ }^{18} \mathrm{~F}-\mathrm{FDG}$ uptake in bowel wall. (C) Coronal reformatted CT enterogram shows abnormal segments with water attenuation by visual assessment in distal ileum (mural enhancement, 135 $\mathrm{HU}$; wall thickness, $11 \mathrm{~mm}$ ).
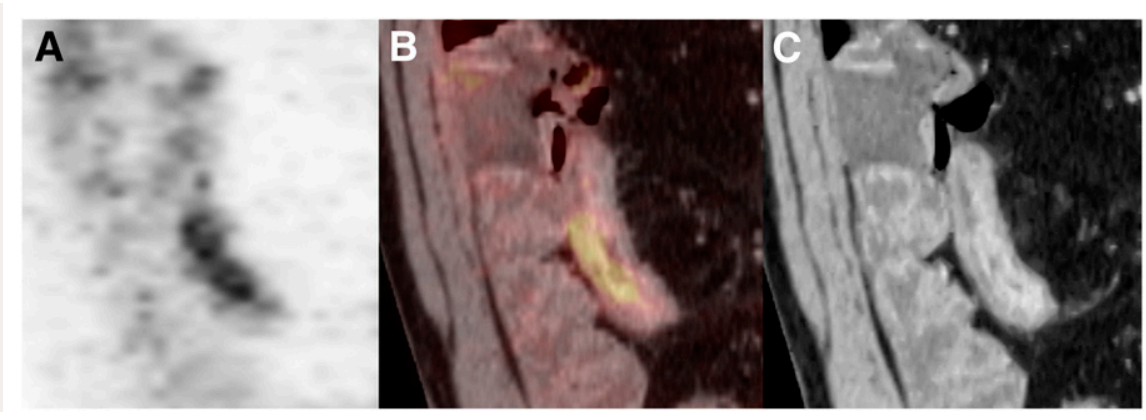

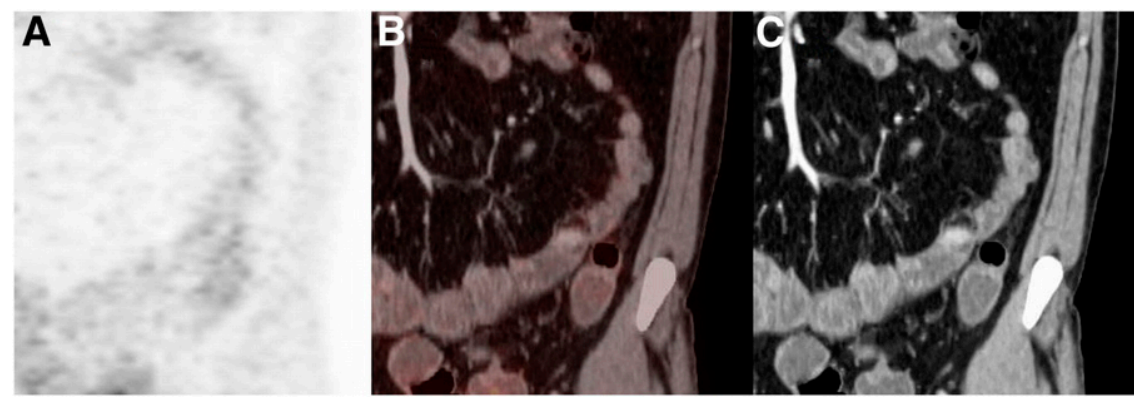

FIGURE 2. Intramural fat attenuation on PET/CT enterography. (A) Coronal PET image shows normal uptake of ${ }^{18} \mathrm{~F}$ FDG in bowel wall (SUVmax, 2.1). (B) Fused PET/CT enterogram shows no uptake in abnormal bowel wall. (C) Coronal reformatted CT enterogram shows abnormal segment with fat attenuation by visual assessment in proximal segment of ileum (mural enhancement, $125 \mathrm{HU}$; wall thickness, $8.5 \mathrm{~mm}$ ).

and $z$ ) planes. The maximum-intensity voxel within this volume of interest, calibrated to SUV normalized for body weight, was recorded as maximal SUV (SUVmax).

\section{Statistical Analysis}

Results are shown as mean $\pm \mathrm{SD}$, with $95 \%$ confidence interval (CI) of the mean. A 2-sided Mann-Whitney test was used to compare quantitative measurements obtained by PET and CT enterography between normal and abnormal bowel segments. CT enterography measurements of mural enhancement and mural thickness were correlated with PET measurements of SUVmax using Spearman rank correlation. The statistical significance of the difference between the CT-enterography- and PET-derived correlation coefficients was determined. Differences in measured SUVmax by PET for intramural attenuation, increased attenuation of the perienteric fat, and the comb sign by CT enterography were assessed using 1-way ANOVA. When there was a significant difference, the Student-Newman-Keuls test for pairwise comparison of subgroups was performed. The $P$ level for statistical significance was set at 0.05. Statistical analyses were performed using MedCalc software (version 11.1.1.0).
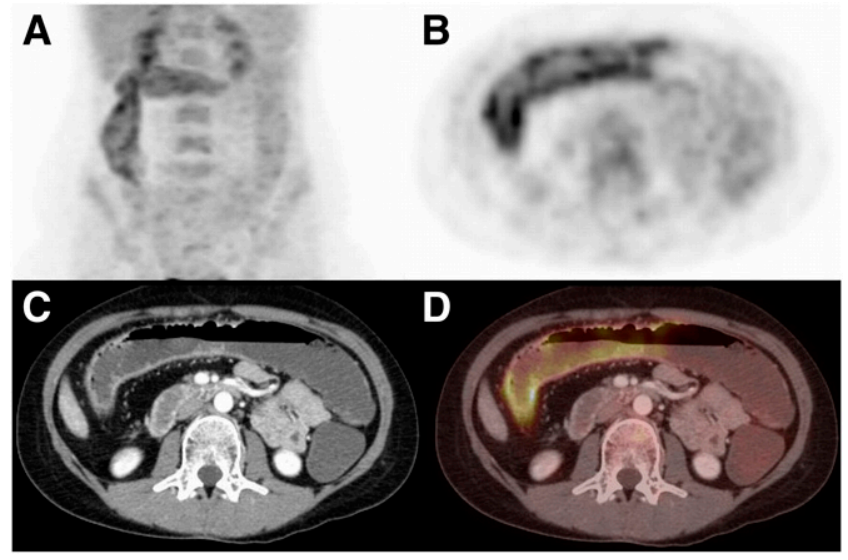

FIGURE 3. Intramural tissue attenuation on PET/CT enterography. (A) PET maximum-intensity-projection image shows abnormal uptake in right hemicolon. (B) Axial PET image shows increased uptake of ${ }^{18} \mathrm{~F}-\mathrm{FDG}$ in bowel wall of right hemicolon (SUVmax, 5.1). (C) Axial reformatted CT enterogram shows abnormal segment of colon with tissue attenuation by visual assessment (mural enhancement, 127 $\mathrm{HU}$; wall thickness, $8.0 \mathrm{~mm}$ ). (D) Fused PET/CT enterogram shows ${ }^{18} \mathrm{~F}-\mathrm{FDG}$ uptake in bowel wall.

\section{RESULTS}

Normal Segments

Twenty-eight segments of normal-appearing ileum and 28 segments of normal-appearing colon were noted. CT enterography wall thickness $(n=56)$ was $1.8 \pm 0.6 \mathrm{~mm}$ (95\% CI, 1.7-2.0 mm); there was no significant difference in the measured thickness between colon and ileum (1.8 \pm 0.7 and $1.8 \pm 0.5 \mathrm{~mm}$, respectively; $P=0.74)$. Mural enhancement $(n=56)$ was $49.9 \pm 14.7$ HU (95\% CI, 45.9$53.8 \mathrm{HU})$; as expected, enhancement was higher in the ileum $(56.6 \pm 13.7 \mathrm{HU})$ than in the colon $(43.1 \pm 12.5 \mathrm{HU}$, $P=0.0003$ ). PET SUVmax was $2.1 \pm 0.69$ (95\% CI, 1.92.2); there was no significant difference in the measured SUVmax between colon and ileum $(2.1 \pm 0.87$ and $1.99 \pm$ 0.44 , respectively; $P=0.46$ ). An example is shown in Figure 4.

\section{Abnormal Segments}

Eighty-five abnormal segments were noted in 22 patients. There were 57 abnormal segments in the ileum and 28 in the colon. Six patients had no abnormal segments noted on CT enterography and no pathologic uptake of ${ }^{18} \mathrm{~F}$ -

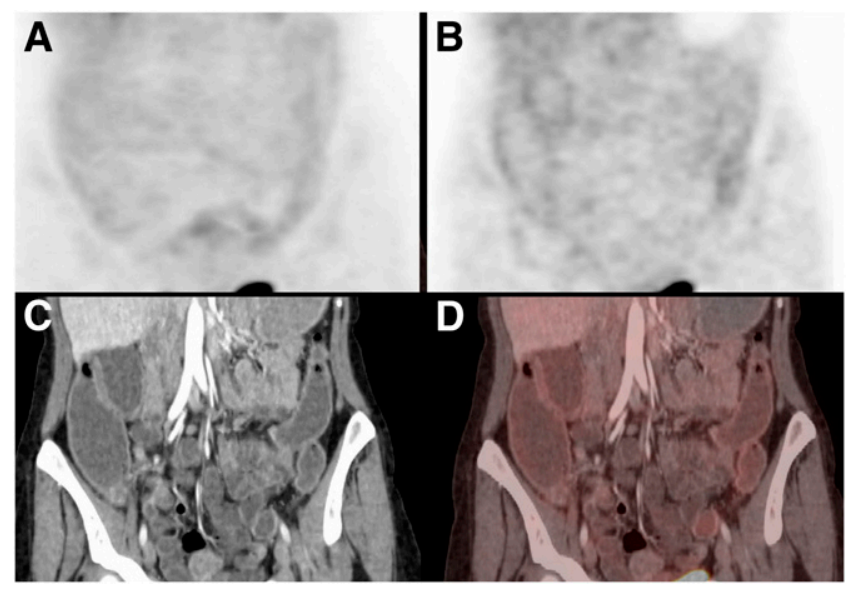

FIGURE 4. Normal findings on PET/CT enterography: PET maximum-intensity-projection image (A); coronal PET image (B); coronal reformatted CT enterogram (C); and fused PET/ CT enterogram (D). Well-distended thin-walled bowel is seen, with uniform enhancement and physiologic uptake of ${ }^{18} \mathrm{~F}-\mathrm{FDG}$ in bowel wall. 
FDG in the bowel: 3 were patients with suspected $C D$, and 3 were patients with previously diagnosed CD.

Mean wall thickness was significantly higher in abnormal segments than in normal segments $(8.6 \pm 2.5 \mathrm{~mm}[95 \% \mathrm{CI}$, $8.1-9.2 \mathrm{~mm}] ; P<0.0001)$. There was no significant difference in wall thickness between segments of ileum and segments of colon $(8.7 \pm 2.5$ and $8.4 \pm 2.5 \mathrm{~mm}$, respectively; $P=0.74)$.

Mean mural enhancement was significantly higher in abnormal segments than in normal segments $(113.4 \pm 23.7$ HU [95\% CI, 108.3-118.5 HU]; $P<0.0001)$. Abnormal mural enhancement was significantly higher in the ileum than in the colon $(117.9 \pm 21.7 \mathrm{HU}$ and $104.2 \pm 25.3 \mathrm{HU}$, respectively; $P=0.013$ ).

SUVmax was significantly higher in abnormal segments than in normal segments $(5.0 \pm 2.5$ [95\% CI, 4.5-5.5]; $P<$ 0.0001). There was no significant difference in SUVmax between segments of ileum and segments of colon (5.1 \pm 2.7 and $4.8 \pm 2.0$, respectively; $P=0.75$ ).

A good correlation was found between SUVmax measurements and wall thickness in the colon and ileum $(r=$ $0.69, P<0.00001$, and $r=0.65, P<0.00001$, respectively) (Supplemental Fig. 3). There was no significant difference between the correlation in the ileum and the correlation in the colon $(P=0.62)$.

A good correlation was found between SUVmax and mural enhancement in the colon and ileum $(r=0.77, P<$ 0.00001 , and $r=0.59, P<0.00001$, respectively) (Supplemental Fig. 3). There was no significant difference between the correlation in the ileum and the correlation in the colon $(P=0.054)$.

Table 1 shows the differences in measured SUVmax between segments with different intramural attenuation by visual assessment. Examples are shown in Figures 1-3. SUVmax was significantly higher in segments with water attenuation than in segments with tissue attenuation, and SUVmax was higher in segments with tissue attenuation than in segments with fat attenuation $(P<0.001)$.

Table 2 shows the differences in measured SUVmax between segments with different levels of perienteric fat infiltration and the comb sign by visual assessment. SUVmax was significantly higher in segments with intense perienteric fat attenuation than in segments with moderate perienteric fat attenuation, and SUVmax was higher in segments with moderate perienteric fat attenuation than in segments without perienteric fat attenuation $(P<0.001)$. SUVmax was significantly higher in segments with an intense and moderate comb sign than in segments without the comb sign $(P<$ $0.001)$. SUVmax in segments with an intense comb sign was similar to that in segments with a moderate comb sign $(P=$ $0.6)$.

\section{DISCUSSION}

Our results demonstrated that SUVmax may be an objective indication of active inflammation in segments of bowel that are abnormal on CT enterography. We combined PET and CT enterography in a single examination and compared the level of ${ }^{18} \mathrm{~F}$-FDG uptake measured by SUVmax with CT enterography patterns of disease in patients with CD. An objective assessment of the grade and severity of disease activity is important in managing and monitoring response to therapy in patients with CD. Disease activity is currently evaluated through clinical indices, endoscopy, radiology, and biochemical markers of inflammation. However, none of these tools provides all the information needed to objectively measure disease activity.

${ }^{18} \mathrm{~F}$-FDG accumulates in areas of active inflammation, and recent studies have suggested that ${ }^{18} \mathrm{~F}-\mathrm{FDG}$ PET/CT, compared with endoscopy, histology, radiography, and smallbowel imaging, is useful in detecting inflamed segments of small and large bowel in inflammatory bowel disease, with sensitivities and specificities ranging from $54 \%$ to $98 \%$ and $55 \%$ to $81 \%$, respectively $(4,7-12)$. However, most of these studies did not completely assess the entire bowel wall. Louis et al. (7) showed that an intense SUV combined with thickened bowel wall in areas of normal endoscopic appearance establishes the diagnosis of active $\mathrm{CD}$. They examined 95 bowel segments and demonstrated a sensitivity of $72.9 \%$ in detecting endoscopic inflammation and a sensitivity of $100 \%$ in detecting severely inflamed segments with deep ulcers and strictures. Das et al. (9) found that combined PET and CT enteroclysis detects significantly more lesions in both the small and the large bowels than does conventional barium studies and colonoscopy in patients with inflammatory bowel disease.

PET/CT is usually performed with positive oral contrast material. CT enterography usually uses negative oral contrast material that causes bowel distention without increasing CT attenuation and helps avoid the potential PET attenuation artifacts that can be seen with high-density barium- or iodinebased oral contrast material. Also, the bowel distension obtained by $\mathrm{CT}$ enterography reduces background ${ }^{18} \mathrm{~F}-\mathrm{FDG}$ activity and physiologic uptake of ${ }^{18} \mathrm{~F}-\mathrm{FDG}$ in the bowel, allowing better definition of uptake in the bowel wall (1416).

The characteristic CT enterography appearances of active $\mathrm{CD}$ are bowel wall thickening, mural hyperenhancement, mural stratification, increased attenuation of the perienteric

\begin{tabular}{|c|c|c|c|c|c|c|}
\hline \multicolumn{3}{|c|}{ Intramural attenuation } & \multirow[b]{2}{*}{ 1-way ANOVA } & \multicolumn{3}{|c|}{ 2-sample $t$ testing } \\
\hline Water & Tissue & Fat & & Water vs. tissue & Water vs. fat & Tissue vs. fat \\
\hline $6.3 \pm 2.5(n=42)$ & $4.3 \pm 1.7(n=29)$ & $2.5 \pm 0.8(n=14)$ & $P<0.001$ & $P=0.0002$ & $P<0.0001$ & $P<0.0001$ \\
\hline
\end{tabular}


TABLE 2. Segmental SUVmax Measurements by Visual Assessment of Perienteric Fat Infiltration and Comb Sign

\begin{tabular}{|c|c|c|c|c|c|c|c|}
\hline \multirow[b]{3}{*}{ Finding } & \multirow{2}{*}{\multicolumn{3}{|c|}{ Visual assessment }} & \multirow{3}{*}{$\begin{array}{c}\text { 1-way } \\
\text { ANOVA }\end{array}$} & \multicolumn{3}{|c|}{2 -sample $t$ testing } \\
\hline & & & & & Absent & Absent & Moderate \\
\hline & Absent & Moderate & Intense & & vs. moderate & vs. intense & vs. intense \\
\hline Fat infiltration & $3.5 \pm 1.9(n=24)$ & $4.9 \pm 1.6(n=46)$ & $7.7 \pm 3.4(n=15)$ & $P<0.001$ & $P=0.002$ & $P=0.0003$ & $P=0.006$ \\
\hline Comb sign & $3.5 \pm 1.5(n=24)$ & $5.5 \pm 2.5(n=48)$ & $5.9 \pm 2.7(n=13)$ & $P=0.001$ & $P=0.0001$ & $P=0.0028$ & $P=0.60^{*}$ \\
\hline
\end{tabular}

fat, and the comb sign $(1-3,5)$. In a recent study, Ahmadi et al. (10) evaluated 48 segments of abnormal bowel in 30 patients by PET/CT enterography. Ahmadi et al. used a CT enterography score based on visual mural enhancement (1, physiologic enhancement; 2 , mild to moderate enhancement; and 3, hyperenhancement) added to bowel thickness measurements $(\mathrm{mm})$ and found a fair correlation $(r=0.31)$ between the CT enterography score and SUVmax. In the present study, we compared objective measurements of mural thickness (mm) and mural enhancement (HU), as well as subjective CT enterography findings associated with active CD. A good correlation was found between the PET SUVmax measurements and the CT enterography mural thickness and enhancement measurements $(P<0.00001)$. Mural hyperenhancement and increased mural thickness have been reported as objective CT enterography findings of active $\mathrm{CD}(17,18)$. However, mural attenuation depends on the contrast material injection protocol, the degree of bowel distension, and the method of measurement. Collapsed bowel segments show increased attenuation, compared with distended bowel segments, and may cause overdiagnosis of inflamed bowel loops (1-3). Also, chronic fibrosis leads to wall thickening and may show increased enhancement ( $1-$ $3,5)$. Therefore, simple morphologic measurements of mural thickening and mural enhancement may not be used as an isolated objective assessment of disease activity in CD.

Subjective assessments of actively inflamed bowel loops on CT enterography include mural stratification, increased perienteric fat attenuation, and the comb sign. Attenuation of the intramural portion of the bowel wall can vary with the level of enteric inflammation: intramural edema (water attenuation) indicates active inflammation, intramural softtissue attenuation may represent an inflammatory infiltrate, and intramural fat usually indicates past or chronic inflammation (1-3). There was a statistically significant difference in mean SUVmax among the 3 patterns of intramural attenuation $(P<0.001)$. Mean SUVmax was highest for water attenuation, followed by tissue attenuation and then fat attenuation.

Increased perienteric fat infiltration and the comb sign are indirect CT enterography findings of inflammation involving the perienteric tissues (1-3). There was a significant difference in SUVmax among the 3 levels of perienteric fat infiltration $(P<0.001)$. SUVmax was higher in segments with an intense increase in perienteric fat infiltration than in segments with a moderate increase, and SUVmax was higher in segments with a moderate increase in perienteric fat infiltration than in segments without an increase.

The comb sign is not sensitive for active CD but is quite specific, correlating with clinically advanced and extensive CD $(1,17)$. SUVmax showed a significant difference among the 3 levels of the comb sign $(P<0.001)$. There was no significant difference in SUVmax between a moderate and intense comb sign $(P=0.6)$. However, SUVmax was significantly higher in segments with the comb sign than in segments without it.

The limitations of our study include the small population and the use of only 1 radiologist to interpret the CT enterograms. On the one hand, the choice of a single radiologist could have biased the detection of abnormal segments, but on the other hand, interobserver agreement for characteristic CT enterography findings in active $\mathrm{CD}$ has been shown to be high (17), and a single skilled, meticulous reader could detect abnormal segments that might be difficult for others to duplicate.

\section{CONCLUSION}

This study showed that SUVmax determination might be a reliable objective method for quantifying the activity and severity of CD. Future studies are warranted to evaluate if combined PET/CT enterography has an important role in the clinical management of $\mathrm{CD}$.

\section{REFERENCES}

1. Paulsen SR, Huprich JE, Hara AKCT. Enterography: noninvasive evaluation of Crohn's disease and obscure gastrointestinal bleed. Radiol Clin North Am. 2007; 45:303-315.

2. Furukawa A, Saotome T, Yamasaki M, et al. Cross-sectional imaging in Crohn's disease. Radiographics. 2004;24:689-702.

3. Paulsen SR, Huprich JE, Fletcher JG, et al. CT enterography as a diagnostic tool in evaluating small bowel disorders: review of clinical experience with over 700 cases. Radiographics. 2006;26:641-662.

4. Halpenny DF, Burke JP, Lawlor GO, O'Connell M. Role of PET and combination PET/CT in the evaluation of patients with inflammatory bowel disease. Inflamm Bowel Dis. 2009;15:951-958.

5. Macari M, Megibow AJ, Balthazar EJ. A pattern approach to the abnormal small bowel: observations at MDCT and CT enterography. AJR. 2007;188:1344-1355.

6. Malide D, Davies-Hill TM, Levine M, et al. Distinct localization of GLUT-1, -3, and -5 in human monocyte-derived macrophages: effects of cell activation. Am J Physiol. 1998;274:E516-E526.

7. Louis E, Ancion G, Colard A, Spote V, Belaiche J, Hustinx R. Noninvasive assessment of Crohn's disease intestinal lesions with ${ }^{18} \mathrm{~F}$-FDG PET/CT. J Nucl Med. 2007;48:1053-1059. 
8. Neurath MF, Vehling D, Schunk K, et al. Noninvasive assessment of Crohn's disease activity: a comparison of ${ }^{18} \mathrm{~F}$-fluorodeoxyglucose positron emission tomography, hydromagnetic resonance imaging, and granulocyte scintigraphy with labeled antibodies. Am J Gastroenterol. 2002;97:1978-1985.

9. Das CJ, Makharia G, Kumar R, et al. PET-CT enteroclysis: a new technique for evaluation of inflammatory diseases of the intestine. Eur J Nucl Med Mol Imaging. 2007;34:2106-2114.

10. Ahmadi A, Li Q, Muller K, et al. Diagnostic value of noninvasive combined fluorine-18 labeled fluoro-2-deoxy-D-glucose positron emission tomography and computed tomography enterography in active Crohn's disease. Inflamm Bowel Dis. November 2, 2009 [Epub ahead of print].

11. Jacene HA, Ginsburg P, Kwon J, et al. Prediction of the need for surgical intervention in obstructive Crohn's disease by ${ }^{18} \mathrm{~F}-\mathrm{FDG}$ PET/CT. J Nucl Med. 2009;50:1751-1759.

12. Spier BJ, Perlman SB, Reichelderfer M. FDG-PET in inflammatory bowel disease. Q J Nucl Med Mol Imaging. 2009;53:64-71.
13. Arslan H, Etlik O, Kayan M, et al. Enterography with lactulose solution: preliminary observations. AJR. 2005;185:1173-1179.

14. Sun XG, Huang G, Liu JJ, Wan LR. Comparison of the effect of positive and negative oral contrast agents on ${ }^{18}$ F-FDG PET/CT scan. Hell J Nucl Med. 2009; 12:115-118.

15. Prabhakar HB, Sahani DV, Fischman AJ, Mueller PR, Blake MA. Bowel hot spots at PET-CT. Radiographics. 2007;27:145-159.

16. Antoch G, Kuehl H, Kanja J, et al. Dual-modality PET/CT scanning with negative oral contrast agent to avoid artifacts: introduction and evaluation. Radiology. 2004;230:879-885.

17. Booya F, Fletcher JG, Huprich JE, et al. Active Crohn's disease: CT findings and interobserver agreement for enteric phase CT enterography. Radiology. 2006; 241:787-795.

18. Bodily KD, Fletcher JG, Solem CA, et al. Crohn's disease: mural attenuation and thickness at contrast-enhanced CT enterography - correlation with endoscopic and histologic findings of inflammation. Radiology. 2006;238:505-516. 